\title{
THE BLACK HOLE MASS AND EXTREME ORBITAL STRUCTURE IN NGC 1399
}

\author{
Karl Gebhardt, ${ }^{1}$ Tod R. Lauer, ${ }^{2}$ Jason Pinkney, ${ }^{3}$ Ralf Bender, ${ }^{4}$ Douglas Richstone, ${ }^{5}$ Monique Aller, ${ }^{5}$ \\ Gary Bower, ${ }^{6}$ Alan Dressler, ${ }^{7}$ S. M. Faber, ${ }^{8}$ Alexei V. Filippenko, ${ }^{9}$ Richard Green, ${ }^{2}$ Luis C. Ho, ${ }^{7}$ \\ John Kormendy, ${ }^{1}$ Christos Siopis, ${ }^{5}$ and Scott Tremaine ${ }^{10}$ \\ Received 2007 April 11; accepted 2007 August 16
}

\begin{abstract}
The largest galaxies, and in particular central galaxies in clusters, offer unique insight into understanding the mechanism for the growth of nuclear black holes. We present Hubble Space Telescope kinematics for NGC 1399, the central galaxy in Fornax. We find the best-fit model contains a black hole of $(5.1 \pm 0.7) \times 10^{8} M_{\odot}$ (at a distance of $21.1 \mathrm{Mpc}$ ), a factor of over 2 below the correlation of black hole mass and velocity dispersion. We also find a dramatic signature for central tangential anisotropy. The velocity profiles on adjacent sides $0.5^{\prime \prime}$ away from the nucleus show strong bimodality, and the central spectrum shows a large drop in the dispersion. Both of these observations point to an orbital distribution that is tangentially biased. The best-fit orbital model suggests a ratio of the tangential to radial internal velocity dispersions of 3 . This ratio is the largest seen in any galaxy to date and will provide an important measure for the mode by which the central black hole has grown.
\end{abstract}

Subject headings: galaxies: general — galaxies: nuclei — galaxies: statistics

Online material: color figures

\section{INTRODUCTION}

It is clear that the mass of the central black hole is related to its host galaxy in a fundamental way. Dressler (1989), Kormendy (1993), Kormendy \& Richstone (1995), and Magorrian et al. (1998) were the first to highlight a correlation between the black hole mass and the bulge light. Subsequently, many other correlations have been found, with the tightest being that between black hole mass and velocity dispersion (Gebhardt et al. 2000; Ferrarese $\&$ Merritt 2000). Numerous theoretical models have been proposed to explain these correlations, and the most compelling to date are those that work through active galactic nucleus (AGN) feedback mechanisms (Silk \& Rees 1998; Fabian 1999; Springel et al. 2005; Robertson et al. 2006). To push further requires more secure observations. The problem is that the uncertainties in the black hole mass estimates are still large (around 30\%-50\%) and, more importantly, the extremes of the correlations are not well explored. We have been targeting the largest galaxies in order to study their central black hole mass. There is only a handful of objects studied with velocity dispersions above $300 \mathrm{~km} \mathrm{~s}^{-1}$.

In this paper we study the giant elliptical NGC 1399, the dominant galaxy in the Fornax Cluster. In addition to providing in-

\footnotetext{
1 Department of Astronomy, University of Texas, Austin, TX 78712; gebhardt@ astro.as.utexas.edu, kormendy@astro.as.utexas.edu.

2 National Optical Astronomy Observatory, P.O. Box 26732, Tucson, AZ 85726; lauer@noao.edu, green@noao.edu.

3 Department of Physics and Astronomy, Ohio Northern University, Ada, OH 45810; j-pinkney@onu.edu.

4 Universitäts-Sternwarte, Scheinerstrasse 1, 81679 Munich, Germany; bender@usm.uni-muenchen.de.

5 Department of Astronomy, University of Michigan, Dennison Building, Ann Arbor, MI 48109; dor@astro.lsa.umich.edu,jpinkney@astro.lsa.umich.edu.

${ }^{6}$ Computer Sciences Corporation, Space Telescope Science Institute, 3700 San Martin Drive, Baltimore, MD 21218; bower@stsci.edu.

7 Observatories of the Carnegie Institution of Washington, 813 Santa Barbara Street, Pasadena, CA 91101; dressler@ociw.edu, lho@ociw.edu.

${ }^{8} \mathrm{UCO} /$ Lick Observatories, University of California, Santa Cruz, CA 95064; faber@ucolick.org.

9 Department of Astronomy, University of California, Berkeley, CA 947203411; alex@astro.berkeley.edu.

${ }^{10}$ Princeton University Observatory, Peyton Hall, Princeton, NJ 08544; tremaine@astro.princeton.edu.
}

formation on the upper end of black hole correlations, central galaxies in clusters offer unique insight. These galaxies are subject to significant accretion and mergers, and it is important to understand whether the black hole grows as the galaxy grows. Houghton et al. (2006) study NGC 1399 using adaptive optics (AO) observations on the Very Large Telescope (VLT); they find a black hole mass of $(1.2 \pm 0.6) \times 10^{9} M_{\odot}$ (for a distance of $19.9 \mathrm{Mpc})$. We find a black hole mass of $(5.1 \pm 0.7) \times 10^{8} M_{\odot}$ (for a distance of 21.1 Mpc, as used in Lauer et al. [2005]). While over a factor of 2 different, we are consistent within $1 \sigma$ (the Houghton et al. result is only a $2 \sigma$ significance for a black hole detection). Furthermore, we find consistent results in terms of the central orbital structure. We use a distance of $21.1 \mathrm{Mpc}$ to NGC 1399 from Tonry et al. (2001), but scaled to $H_{0}=70$ as in Lauer et al. (2005).

\section{DATA}

\subsection{HST Observations}

The surface brightness profile comes from Wide Field Planetary Camera 2 (WFPC2) observations for Hubble Space Telescope (HST) programs GO-5990 and GO-8214 (PI: Grillmair). NGC 1399 was observed for $4000 \mathrm{~s}$ in F606W and for $5200 \mathrm{~s}$ in F450W, with the galaxy centered on the PC. The surface brightness, ellipticity, and color gradient profiles are shown in Figure 1. Due to NGC 1399 being nearly round, the position angle is very uncertain and we do not include discussion of it. The reductions are discussed by Lauer et al. (2005). From its surface brightness profile, NGC 1399 is classified as a core galaxy with a break radius $3.2^{\prime \prime}$ and $\gamma=0.12$ (approximately the central projected density slope), from a Nuker law fit (Lauer et al. 2005). For the surface brightness beyond the HST image, we use ground-based imaging from Saglia et al. (2000). We match the ground-based $R$-band data to the HST surface brightness in the overlap region.

Figure 1 shows a variation in the ellipticity inside of $0.3^{\prime \prime}$. However, since the surface brightness is not steep in the central regions and the isophotes are nearly round, there are large uncertainties in the ellipticities. Thus, the variation could be due to noise, and a constant ellipticity model provides nearly identical residuals. 


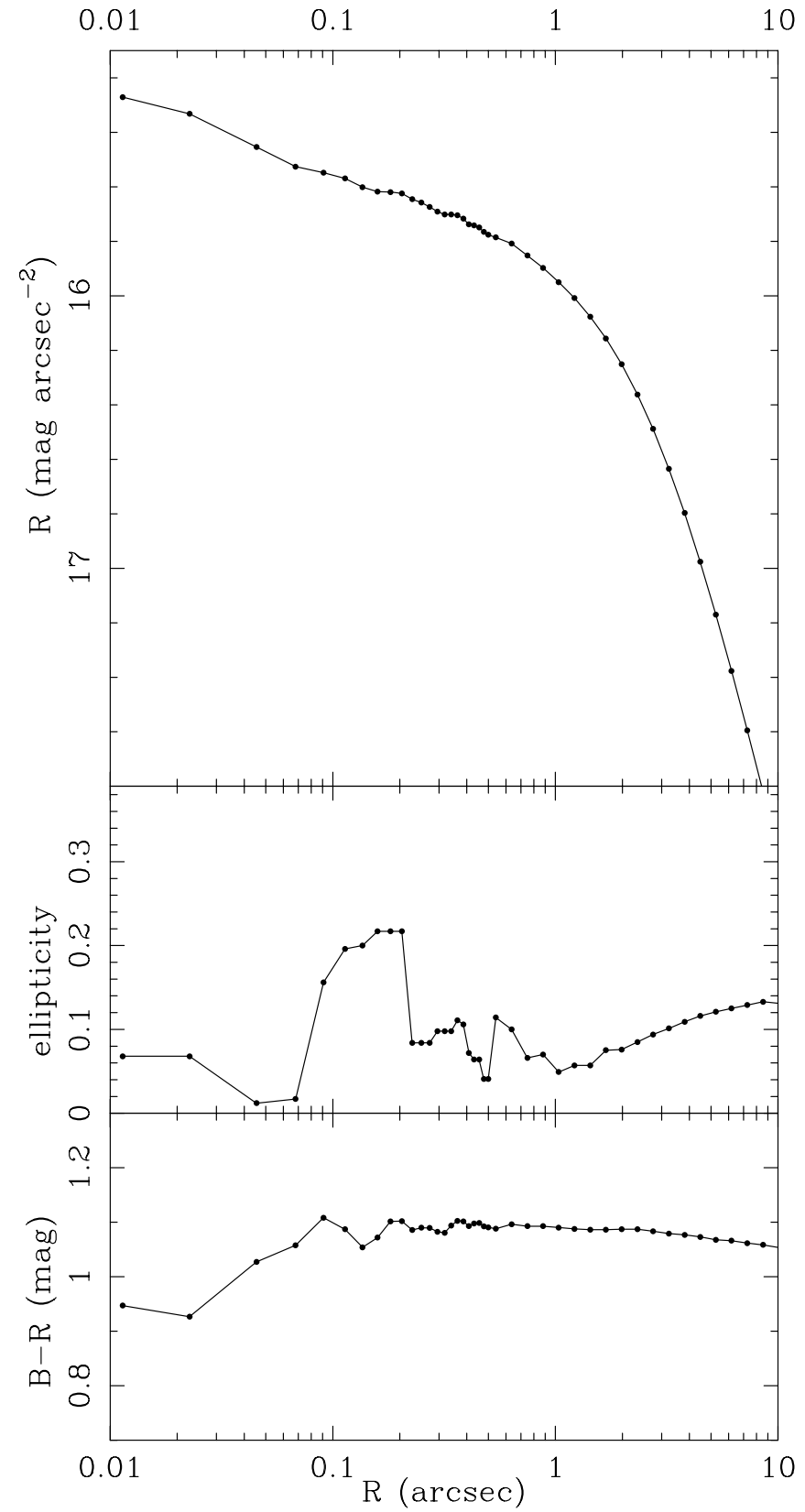

FIG. 1.- HST photometry of NGC 1399. The top panel is the F606W (close to $R$ band) surface brightness profile. We only show the radial region included in HST images. The middle panel is the ellipticity profile. The bottom panel is the difference in surface brightness between F450W (close to the $B$ band) and F606W. The spatial resolution is about $0.05^{\prime \prime}$, so the two central points are within the resolution element; given the shallow gradient of NGC 1399, we expect the central two points to not be biased. There are large ellipticity changes inside of $1^{\prime \prime}$, which is a result of the shallow gradient of the surface brightness and relatively low ellipticity.

In the models that follow, we use a constant ellipticity of 0.1 , but the results do not change much when using an ellipticity of zero. This is also consistent with the surface brightness at larger radii. The position angle is $110^{\circ}$ (measured north to east), and we assume it to be constant. With both a constant position angle and ellipticity, we deproject NGC 1399 as in Gebhardt et al. (1996). This deprojection is used in the dynamical models. From the bottom panel in Figure 1, there is essentially no color gradient in NGC 1399. Thus, we use a constant mass-to-light ratio for the stellar potential. In addition, the color map is also constant as a function of position angle.
We have checked whether the isophotal centers change as a function of radius. This check is important for the discussion in $\S 4$. We find that the center from isophotes at $10^{\prime \prime}$ compared to that derived from the isophotes in the central regions is consistent to within 0.2 pixels, or better than $0.01^{\prime \prime}$. Thus, there appears to be no deviation in the galaxy center. In addition, we find no evidence in the residual map (Lauer et al. 2005) for any second component. Houghton et al. (2006) see an elongation in the central $0.5^{\prime \prime}$, suggesting a possible eccentric disk. We find no such structure in our images; furthermore, the HST images have been subsampled and deconvolved, giving a FWHM around $0.05^{\prime \prime}$, better than the $0.078^{\prime \prime}$ as reported for the AO $K$-band image of Houghton et al. (2006). Still, it is difficult to reconcile the differences; we attribute them to either different structure in $K$ band versus the $R$ band (however, this is unlikely) or an AO artifact. Higher signal-to-noise ratio $(\mathrm{S} / \mathrm{N})$ and repeat $\mathrm{AO}$ observations will likely help determine the cause.

The central surface brightness of NGC 1399 is $V=16.0 \mathrm{mag}$ $\operatorname{arcsec}^{-2}$, making it one of the faintest targets we have observed with the HST Space Telescope Imaging Spectrograph (STIS) (Pinkney et al. 2003). For our previous observations, however, we have used a high-resolution grating centered on the Ca II triplet region at $8500 \AA$. This region is the best to use since it is not greatly influenced by stellar template mismatch and continuum estimation, as are the bluer regions (Barth et al. 2002). However, the exposure times become prohibitive for targets fainter than $V=16.0 \mathrm{mag}$, since it typically requires exposure times of longer than $17 \mathrm{hr}$ to obtain adequate signal. Complicating the kinematic estimate is that these large galaxies tend to have large black hole masses, and therefore large central dispersions. The dispersion of the central STIS pixel for NGC 4649 is over $600 \mathrm{~km} \mathrm{~s}^{-1}$; given the relatively small equivalent widths of the $\mathrm{Ca}$ II triplet lines, the large dispersion makes the lines almost disappear into the continuum. Our strategy for NGC 1399 is to use a lower resolution grating over the $\mathrm{Ca}$ II $\mathrm{H}$ and $\mathrm{K}$ region, where the lines remain clear even when the dispersion is that high (Dressler 1984).

We obtained $6.67 \mathrm{hr}$ of observations on STIS (Woodgate et al. 1998 ) using the G430L grating with $52^{\prime \prime} \times 0.2^{\prime \prime}$ slit align at $117^{\circ}$ (along the major axis; see Fig. 3 of Lauer et al. 2005). The wavelength range is $2880-5690 \AA$, with $2.746 \AA$ pixel $^{-1}$. We binned on chip by two, providing 0.1 arcsec pixel ${ }^{-1}$ in the spatial direction. This setup gave us $\mathrm{S} / \mathrm{N} \approx 20 \mathrm{pixel}^{-1}$ in the central regions, and the same $\mathrm{S} / \mathrm{N}$ at a radius of $1.6^{\prime \prime}$ by binning over 10 pixels $\left(1.0^{\prime \prime}\right)$.

With the low spectral resolution and the wide slit, we have to pay special attention to the change in the instrumental resolution when observing a point source compared to observing a diffuse source. We observed three different template stars with this setup: HD 141680 (a G8 III star), HD 165760 (G8 III), and HD 188056 (K3 III). We stepped each star perpendicularly across the slit to monitor the change in velocity centroid. The goal was to create a template star that represents the actual surface brightness of the galaxy across the slit. Figure 2 shows the shift in the velocity centroid as a function of position in the $0.2^{\prime \prime}$ and the $0.1^{\prime \prime}$ slit. The peak-to-peak variation is about $700 \mathrm{~km} \mathrm{~s}^{-1}$ for the $0.2^{\prime \prime}$ slit, which is expected given the $2.746 \AA 0.05 \mathrm{arcsec}^{-1} \mathrm{pixel}^{-1}$. Figure 3 plots the relative intensity variation across the slit. This intensity variation must be taken into account as well when creating a proper template; at the edges of either slit, there is almost a $50 \%$ drop in intensity compared to the center.

As a first step, we need to know the actual spectral resolution for our setup and galaxy. Fortunately, for two of the stars, HD 141680 and HD 165760, high-resolution ground-based spectra exist over our spectral range (Leitherer et al. 1996). Therefore, we can compare the high-resolution spectra with our spectra to 


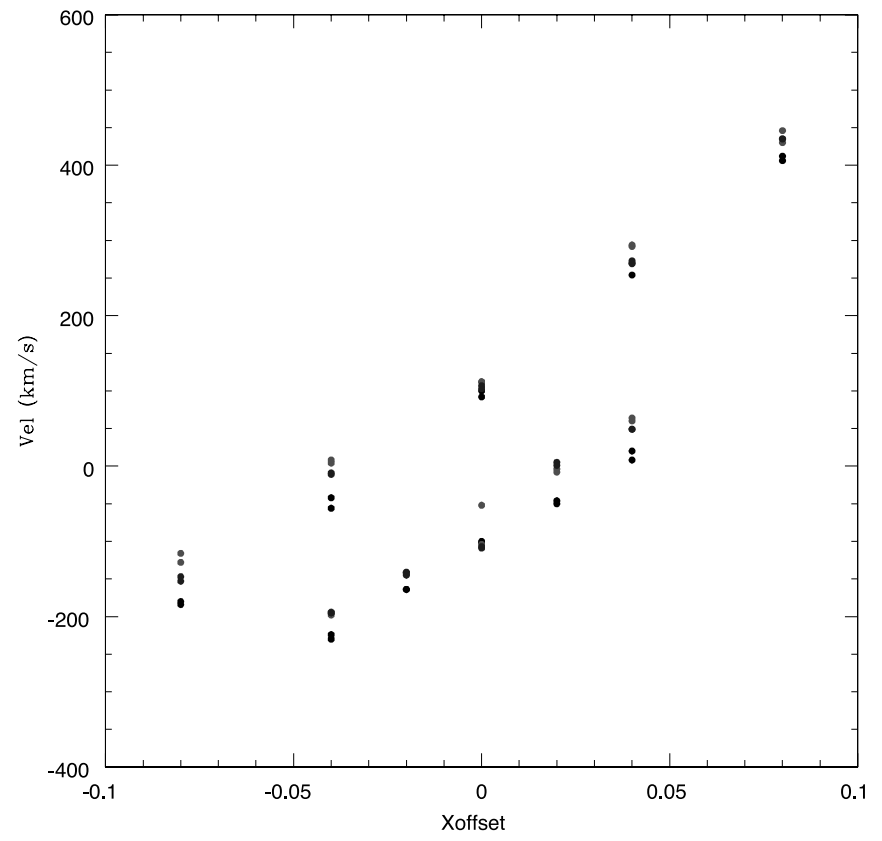

FIG. 2.-Velocity offset as a function of position across the $0.2^{\prime \prime}$ slit (top set of points) and across the $0.1^{\prime \prime}$ slit (bottom set of points). The overall velocity offset is arbitrary and has been set so that the two sets of points do not overlap. Each color corresponds to a different star, and each star was stepped two times across the slit. The slight velocity differences at a given spatial position are a function of both the accuracy in centroiding the velocity and the accuracy in pointing the telescope. The total velocity shift from end to end is as expected, given the $2.746 \AA \mathrm{pixel}^{-1}$ and $0.05^{\prime \prime}$ pixels. [See the electronic edition of the Journal for a color version of this figure.]

obtain the instrumental resolution. Since our wavelength region of interest is $3900-4500 \AA$, we concentrate on this region only. We do this in three different ways to demonstrate the extremes of the results. First, before summing the stepped template, we remove the velocity shift across the slit, which corresponds to a point source. In this case we find that the instrumental $\sigma_{i}=$ $207 \mathrm{~km} \mathrm{~s}^{-1}$, which is roughly what we would expect given this setup. Second, we sum the light for the templates without removing the velocity shift. This case corresponds to a flat source across the slit, and here we find an instrumental $\sigma_{i}=272 \mathrm{~km} \mathrm{~s}^{-1}$. In the third case, we include the surface brightness profile for NGC 1399. There, the light at the edges drops by $20 \%$, and we find an instrumental $\sigma_{i}=275 \mathrm{~km} \mathrm{~s}^{-1}$, consistent within the uncertainties with the flat profile. We can also use the lamp lines to get an estimate of the instrumental dispersion. For lines in this wavelength region, we measure an instrumental $\sigma_{i}=298 \mathrm{~km} \mathrm{~s}^{-1}$. However, in the regions of interest, finding isolated lines is difficult, and $\sigma$ is somewhat overestimated; also, the lamp lines are a completely flat source unlike NGC 1399. Thus, we use $275 \mathrm{~km} \mathrm{~s}^{-1}$ as our instrumental $\sigma_{i}$ (implying a FHWM of $646 \mathrm{~km} \mathrm{~s}^{-1}$ ). Given the instrumental resolution of $275 \mathrm{~km} \mathrm{~s}^{-1}$ for this setup, it is difficult to obtain an accurate estimate of the galaxy dispersion if it is below this.

The spectra cover the range 2880-5690 $\AA$; however, for the kinematics we use only the region $3850-4400 \AA$, covering the $\mathrm{Ca}$ II $\mathrm{H}$ and $\mathrm{K}$ lines and the $\mathrm{G}$ band at $4300 \AA$. Below $3850 \AA$, the lines are weak and the continuum drops, making the $\mathrm{S} / \mathrm{N}$ too low to be useful. The $\mathrm{Mg}$ region around $5100 \AA$ still has good signal, but there are issues with template mismatch that are difficult to overcome. Since NGC 1399 has one of the largest dispersions, it will also have one of the largest equivalent widths for $\mathrm{Mg}$, making it difficult to find templates that accurately reflect the galaxy. This is a long-standing problem, and the traditional

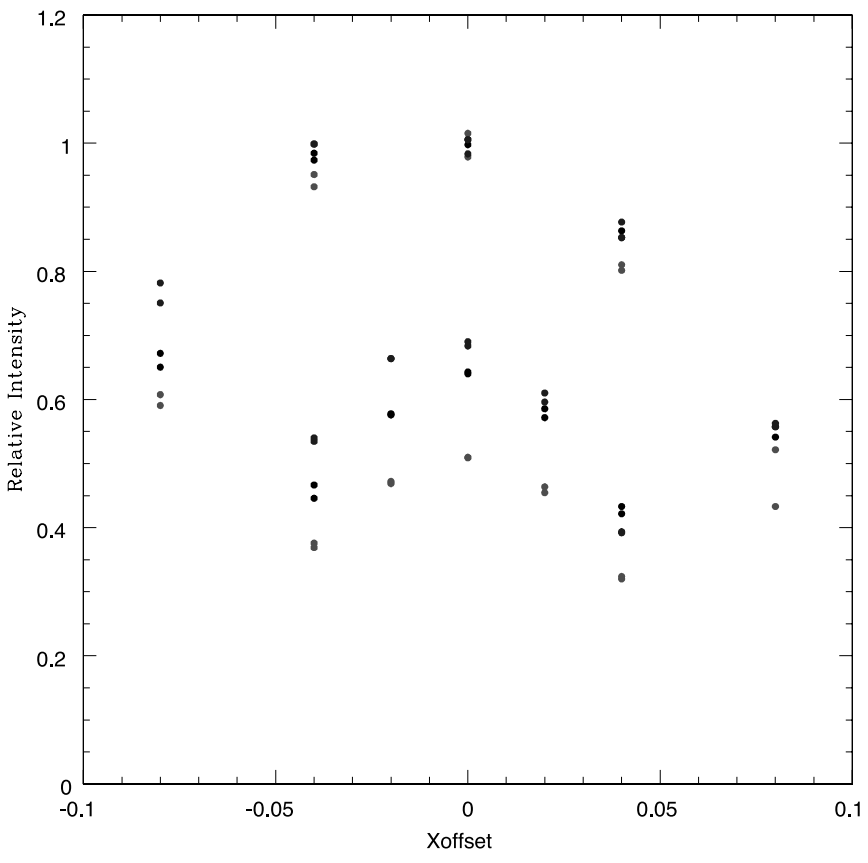

FIG. 3.- Relative intensity as a function of position across the $0.2^{\prime \prime}$ slit (top set of points) and across the $0.1^{\prime \prime}$ slit (bottom set of points). Each color corresponds to the same star as in Fig. 1. [See the electronic edition of the Journal for a color version of this figure.]

method for handling this is to either fit the kinematics in Fourier space (which removes the equivalent width difference), or dilute the galaxy equivalent width by adding a constant to the continuum. Unfortunately, both of these seek to simply match the equivalent width, and any shape difference between the galaxy and the template may manifest itself by biasing the kinematics. We therefore choose to exclude the $\mathrm{Mg}$ region during the fits. Barth et al. (2002) find a similar result when comparing kinematic results from different spectral regions.

Figure 4 plots the spectra of the central pixel and at a radius of $0.8^{\prime \prime}$ for NGC 1399. The two obvious features are the $\mathrm{Ca}$ II $\mathrm{H}$ and $\mathrm{K}$ lines at $3900 \AA$ and the $\mathrm{G}$ band at $4300 \AA$. The template that we use is a result of the procedure described above. For each of the three template stars, we sum the light as it was stepped across the slit with a weight that corresponds to the light profile for NGC 1399. The fit to the galaxy spectrum then involves a convolution with a velocity profile and a linear combination of the three templates. We use the fit as described by Gebhardt et al. (2000) and Pinkney et al. (2003), where we estimate a nonparametric lineof-sight velocity profile. The gray lines in Figure 4 represent the best-fit velocity profiles convolved with the template.

We then extract spectra at different spatial positions. The radii of the extractions (in arcseconds) are 0.0, 0.10, 0.20, 0.30, 0.46, 0.81 , and 1.62. Figure 5 plots the first and second moments as a function of position along the slit. We have extracted the kinematics using both the maximum penalized likelihood and using a Fourier cross-correlation quotient technique (FCQ; Bender 1990). Both sets of points are shown in Figure 5, and the results are similar. There are a few positions where the differences are larger than statistical. For example, at $+0.8^{\prime \prime}$, we find significant differences in the dispersion measured between both techniques. This difference is understandable given the double-peaked nature of the lineof-sight velocity distribution (LOSVD) that we discuss in $\S 3$. When the LOSVD is not unimodal, the way in which the velocity centroid is measured can be very sensitive to fitting procedure. For those positions where the velocity profile is unimodal, the 


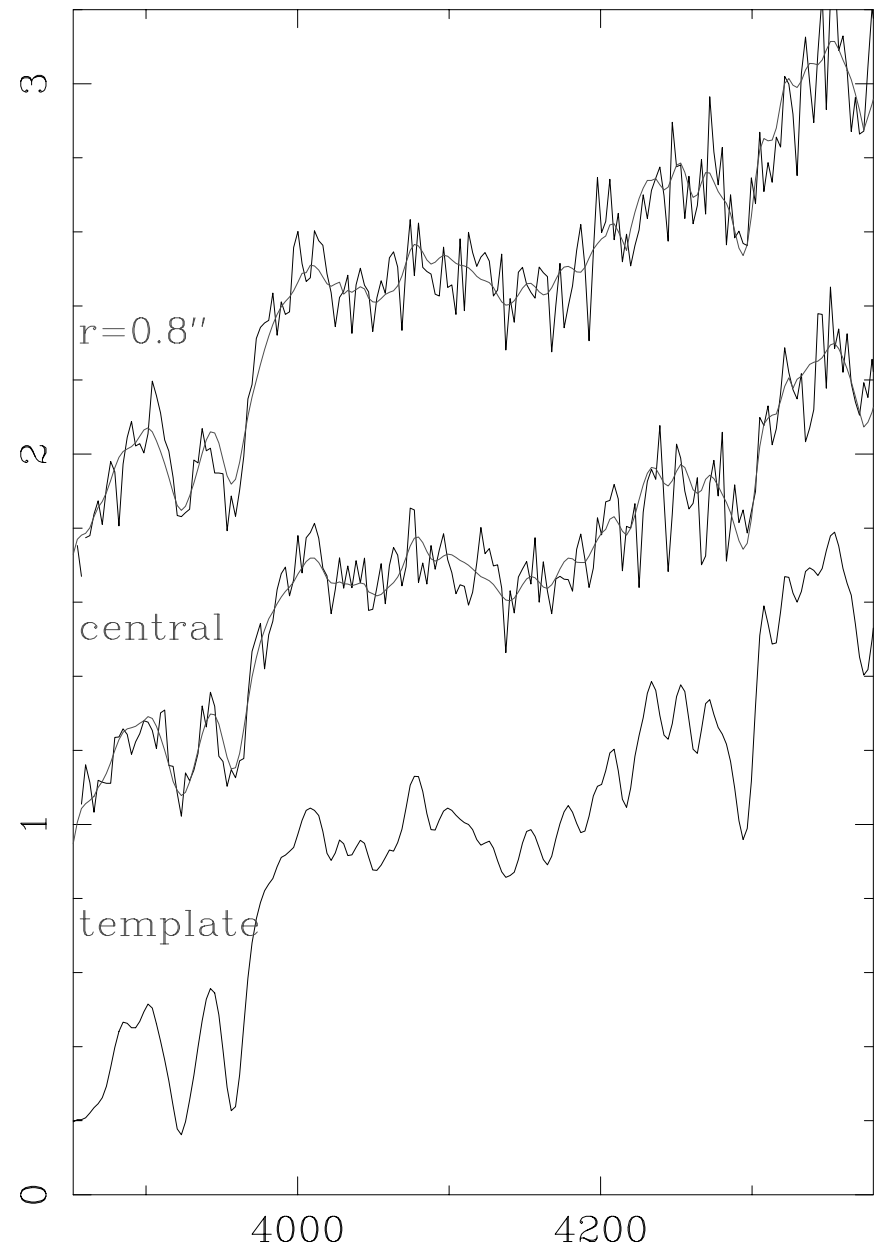

FIG. 4. - Central and radius $=0.8^{\prime \prime}$ spectra for NGC 1399 (two upper black lines) and the template convolved with the best-fit velocity profiles (two gray lines). The bottom spectrum is the template. [See the electronic edition of the Journal for a color version of this figure.]

two techniques agree well. In Figure 5 we also include a symmetrized set of points (solid line). Since the galaxy models that we use are axisymmetric, in order to provide the highest $\mathrm{S} / \mathrm{N}$ we symmetrize the fit to the velocity profile. Details of this symmetrization are given in Pinkney et al. (2003). We use the symmetrized values in the dynamical models.

One sees a dramatic increase in the dispersion to about $500 \mathrm{~km} \mathrm{~s}^{-1}$ at $0.5^{\prime \prime}$ on both sides of the galaxy. The dispersion then drops toward the center to about $270 \mathrm{~km} \mathrm{~s}^{-1}$, which is around the instrumental sigma. However, by just using the dispersion alone, one does not get the complete picture. At $0.5^{\prime \prime}$, the velocity profile shows a dramatic double peak. Figure 6 shows the velocity profiles as determined by a symmetrized fit to opposite sides of the galaxy at the labeled radii. As one moves to larger or smaller radii, the double-hump feature at $r=0.5^{\prime \prime}$ slowly goes away until one gets a nearly Gaussian profile. Furthermore, the double hump appears on both sides of the center at $r=0.5^{\prime \prime}$. Thus, the feature appears to be robust.

This feature and the drop in the dispersion toward the center suggest an orbital distribution strongly dominated by tangential orbits. If the orbital distribution consisted of stars only on circular orbits (but with random orientations), then as one gets closer to the center, the measured projected dispersion would drop to zero, since all orbits would have no radial component. Obviously, a disk is one mechanism that would cause a drop in the central dispersion; but if the disk is seen edge-on, there would be an ob-

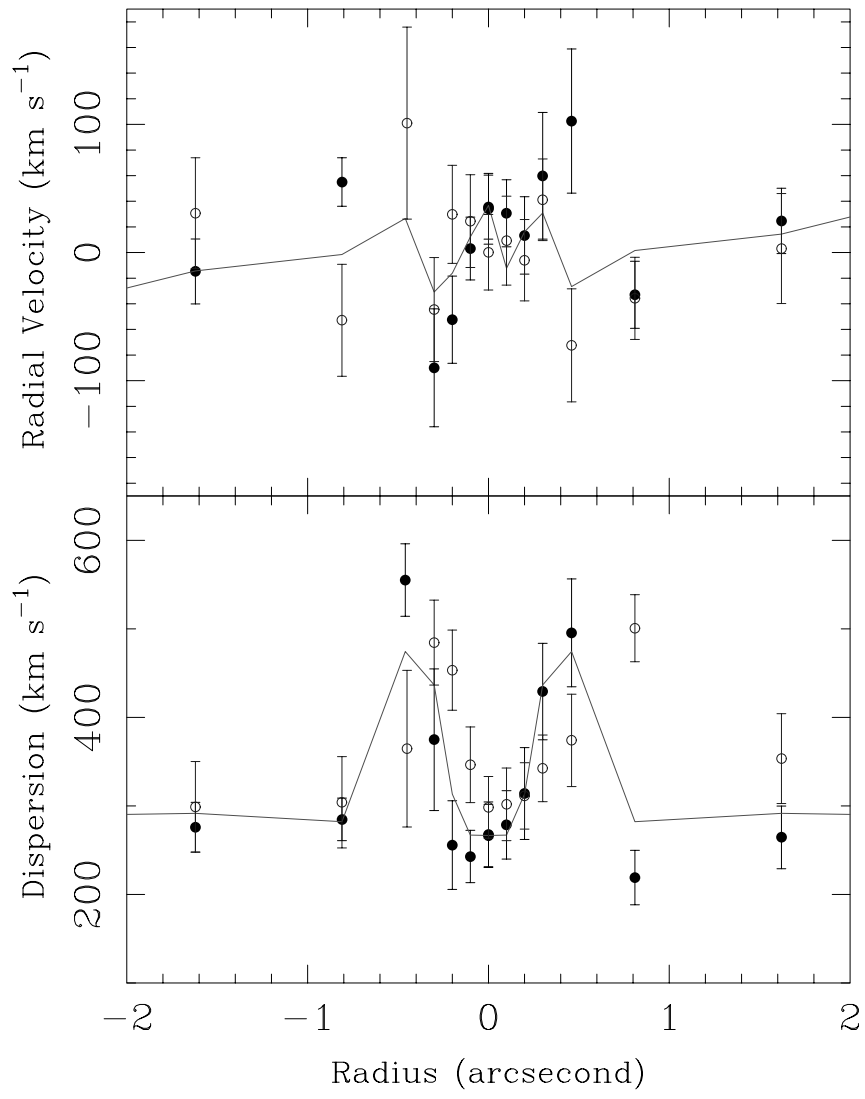

FIG. 5.-First two moments of the velocity profile as a function of position along the slit for NGC 1399. The points with uncertainties are unsymmetrized. The filled circles come from the nonparametric estimate of the velocity profile, and the open circles come from FCQ (Bender 1990). The solid line is a symmetrized version of the kinematic measurements that are used in the dynamical modeling. [See the electronic edition of the Journal for a color version of this figure.]

vious signature in the rotation, which is not seen. If the disk is face-on, it would be nearly impossible for that configuration to cause the double-humped nature seen in the LOSVDs. We are left to conclude that a stellar disk cannot be the cause for the central dispersion drop. However, one can also obtain a drop in the dispersion depending on the shape of the mass density profile. But given the double-humped nature of the velocity profile farther out, it is likely that NGC 1399 is dominated by tangential orbits near the center. In fact, the dynamical models discussed below show the need for tangential orbits. Gebhardt et al. (2003) discuss the signature of tangential orbits seen in other galaxies. Thus, it appears that the largest galaxies, and hence those with the flattest central density profiles, show the strongest amount of tangential bias in the orbital distribution, with NGC 1399 being an extreme example of this phenomenon.

Detailed inspections of the HST image and residual image (Lauer et al. 2005) show nothing at $r=0.5^{\prime \prime}$. As stated previously, Houghton et al. (2006) find a flattened component in their AO $K$-band image, with a similar radial extent. Clearly, additional high-quality images would be worthwhile.

\subsection{Ground-based Spectra}

There are ground-based spectra from two sources. Saglia et al. (2000) published detailed models of NGC 1399 based on longslit data and include Gauss-Hermite polynomial expansion up to h4. Graham et al. (1998) also provide long-slit data with measures of the first two moments only. The comparison between the two data sets is excellent, and there is little difference in which 


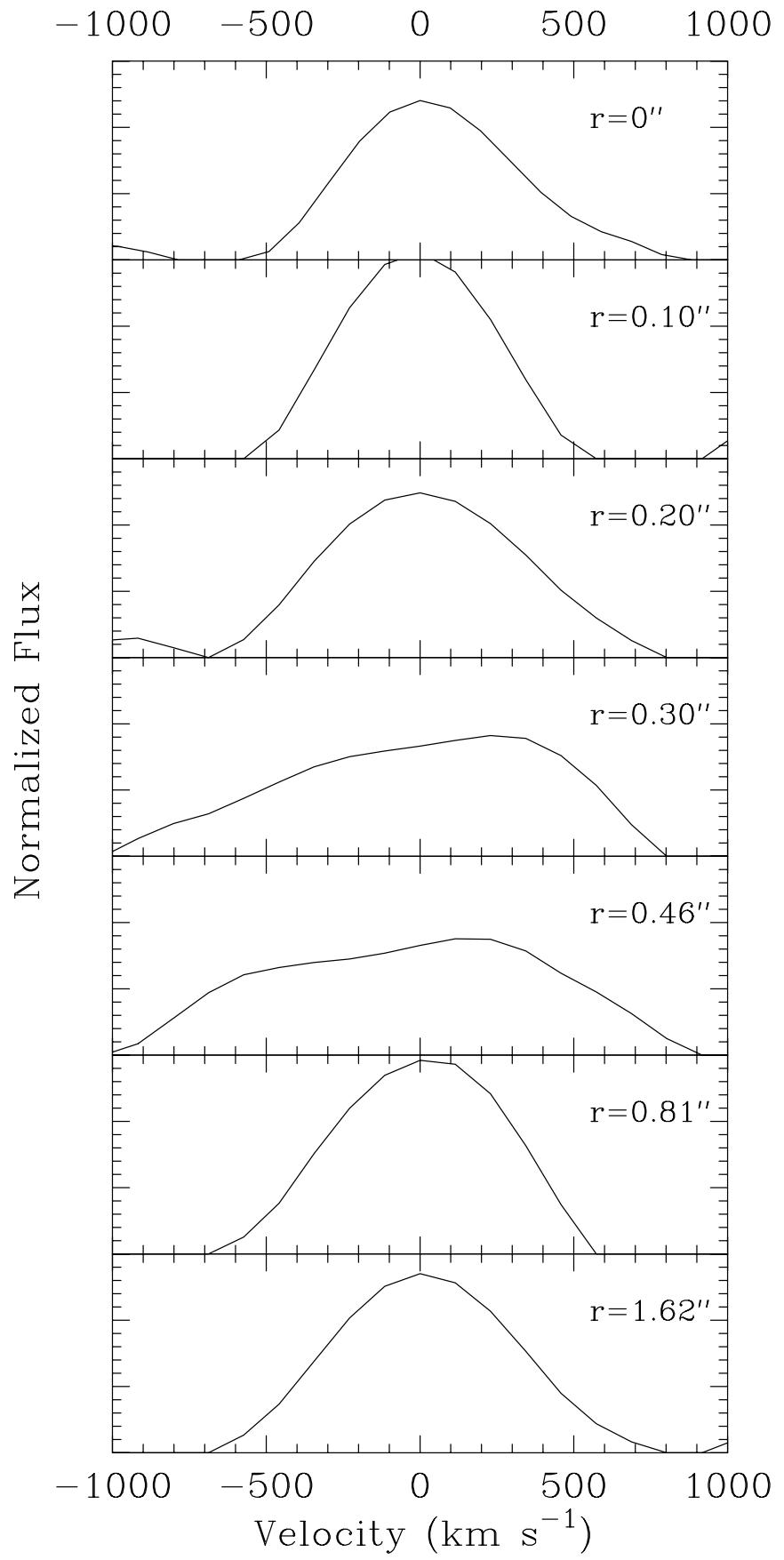

FIG. 6.-Line-of-sight velocity distributions from STIS. These LOSVDs are the fit to both sides of the galaxy at the specified radii; however, the LOSVD is flipped about the systemic velocity, for the opposite side (i.e., this uses the axisymmetric assumption). From $0.3^{\prime \prime}$ to $0.5^{\prime \prime}$, the velocity profile becomes doublepeaked, which is the reason for the increase in the measured second moment at this location in Fig. 5.

one is used for the dynamical models. However, Saglia et al. report additional information on the shape of the velocity profile (four moments compared to the two moments of Graham et al.). Since part of our goal is to constrain the stellar orbital structure, it is better to use as much velocity profile shape information as possible. Thus, for the following analysis, we use only the Saglia et al. data set.

\section{DYNAMICAL MODELS}

The dynamical models that we use are based on orbit superpositions. These are described in detail by Gebhardt et al. (2003)

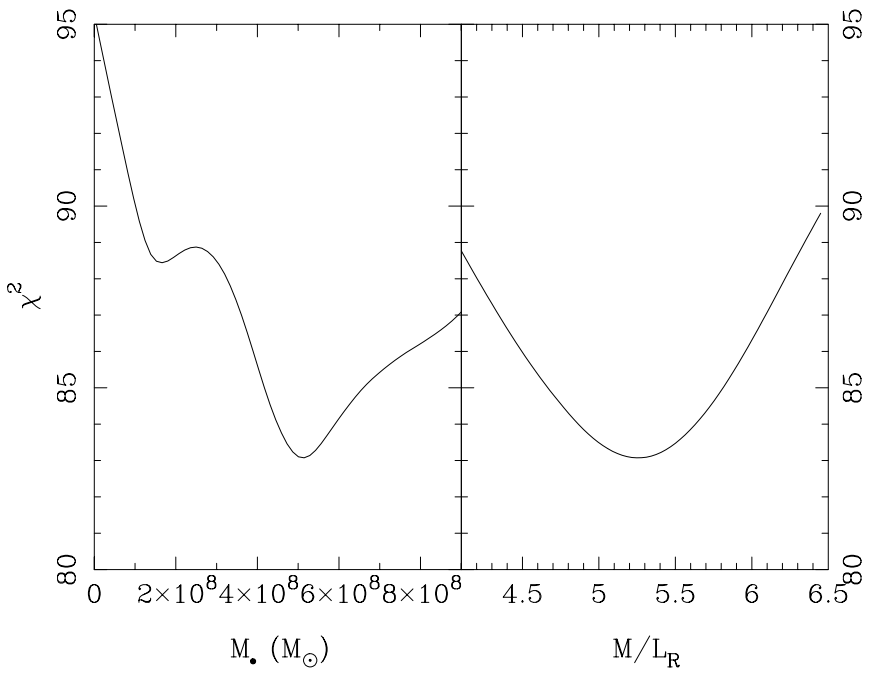

Fig. 7.- The $\chi^{2}$ vs. black hole mass marginalized over $M / L$ (left) and vs. $M / L$ marginalized over black hole mass (right). The total number of parameters used in the fit is 131, but due to the correlation between LOSVD bins, the effective number is smaller.

and Thomas et al. (2004, 2005). We will therefore not discuss these models here, other than to provide our model parameters for NGC 1399. Complete discussions of similar models are also given by Cretton et al. (1999), Verolme et al. (2002), Valluri et al. (2004), and Cappellari et al. (2006).

The models that we use for NGC 1399 have 20 radial and 5 angular bins. Our orbit sampling has 20 energy bins, 40 angular momentum bins (in the $z$-direction), and at least 15 bins for the third integral (see Thomas et al. [2005] for a full description of the orbit sampling). We only need to run models with one sign of angular momentum and then double the number of orbits by flipping the individual velocity profiles about zero velocity. The total number of orbits we have for each orbit library is around 10,000. This orbit library is 2 times higher than we generally use. However, we see no difference in the results when using the smaller library.

The free parameters in the models are black hole mass, massto-light ratio profile, and inclination. We use an edge-on projection for the models shown below. Previous analysis (Gebhardt et al. 2003) shows that different projections have little effect on the black hole mass. Furthermore, since NGC 1399 is nearly round, one could even consider spherical models (as in Houghton et al. 2006), which would minimize projection effects. Figure 7 plots the $\chi^{2}$ versus black hole mass, marginalized over the mass-tolight ratio. There is a well-defined minimum, and we exclude the zero black hole mass with a $\Delta \chi^{2}=20$ (or $>99 \%$ ). Since we marginalize over mass-to-light ratio, our $1 \sigma(68 \%)$ confidence limit corresponds to $\Delta \chi^{2}=1.0$. Thus, we find a best-fit black hole mass of $(5.1 \pm 0.7) \times 10^{8} M_{\odot}$ (marginalized over $\left.M / L\right)$ and the best-fit $M / L_{R}$ of $5.2 \pm 0.4$ (marginalized over black hole mass). In Figure 8 we plot the two-dimensional $\chi^{2}$ contours for black hole mass and mass-to-light ratio.

Figure 9 plots the comparison between the first four GaussHermite coefficients of the data and the models. This plot can only be used for a visual examination of how well the data are fitted, and a statistical evaluation requires comparison with $\Delta \chi^{2}$, as in Figures 7 and 8 . Furthermore, the models are fitted using more information than shown in Figure 9, since we fit the full LOSVDs as opposed to Gauss-Hermite coefficients. This fitting is especially important for NGC 1399, since the LOSVDs are significantly nonGaussian. In Figure 9 we plot three models: our best-fit model, a model with no black hole, and a model with twice the best-fit 


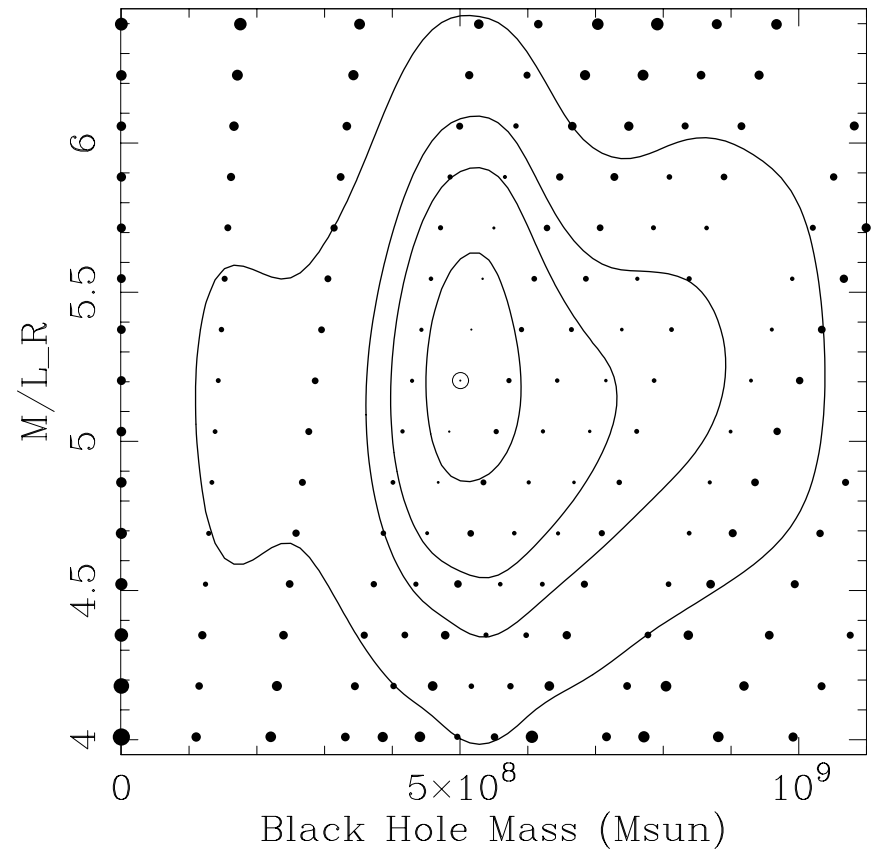

FIG. 8.- Contours of $\chi^{2}$ as a function of black hole mass and mass-to-light ratio. Each point represents a particular model. The contours represent the $68 \%$, $90 \%, 95 \%$, and $99 \%$ confidence for 1 degree of freedom, implying $\Delta \chi^{2}=1.0$, $2.7,4.0$, and 6.6. The circled point is the model that has the minimum value.

mass (so at $10^{9} M_{\odot}$ ). The $\chi^{2}$ difference of the two models compared to the best-fitted model is $10-12$.

Using the integrated dispersion along the major axis out to an effective radius (from the Saglia et al. 2000 data) provides a dispersion of $337 \mathrm{~km} \mathrm{~s}^{-1}$. The black hole mass in NGC 1399 is about a factor of 2.5 below that expected from the BH- $\sigma$ correlation.

The strong tangential anisotropy seen in NGC 1399 is among the most extreme seen in any galaxy to date. It is already clear in Figure 5 that tangential orbits dominate, but we also have a measure from the dynamical models. Figure 10 plots the radial to tangential dispersions as a function of radius for all position angles in the galaxy. From radii $0.1^{\prime \prime}$ to $0.5^{\prime \prime}$, the model becomes highly tangential, with the ratio of the internal dispersions of the radial and tangential components $\sigma_{r} / \sigma_{t}$ around 0.3 . We can compare the NGC 1399 orbital structure to those presented in Gebhardt et al. (2003). There are many galaxies that have this amount of tangential anisotropy in the central region, but none have such a large radial extent. In fact, NGC 1399 is unique in that the central bin is isotropic - which is rare in the Gebhardt et al. sample but then quickly becomes tangential outside the center.

There is important information from the position angles where we do not have data. Even though there are no kinematic constraints there, these offset axes have an effect in projection on the major-axis kinematics, in particular near the center. Thus, there are indirect kinematic constraints. Figure 10 shows that the orbital structure along these offset axes show a structure very similar to that along the major axis.

Houghton et al. (2006) find similar results for the orbital structure. Their ratio of radial to tangential dispersions is plotted as the solid gray line in Figure 10. In both panels, we only plot the ratio for their best-fit model, which has a black hole of $1.2 \times 10^{9} M_{\odot}$. While they do not find the extreme amount of tangential anisotropy that we find for our best-fit model, the trend is very similar. Given the better spatial information for the kinematics in our data, it is not a surprise that we find a stronger change in the anisotropy.

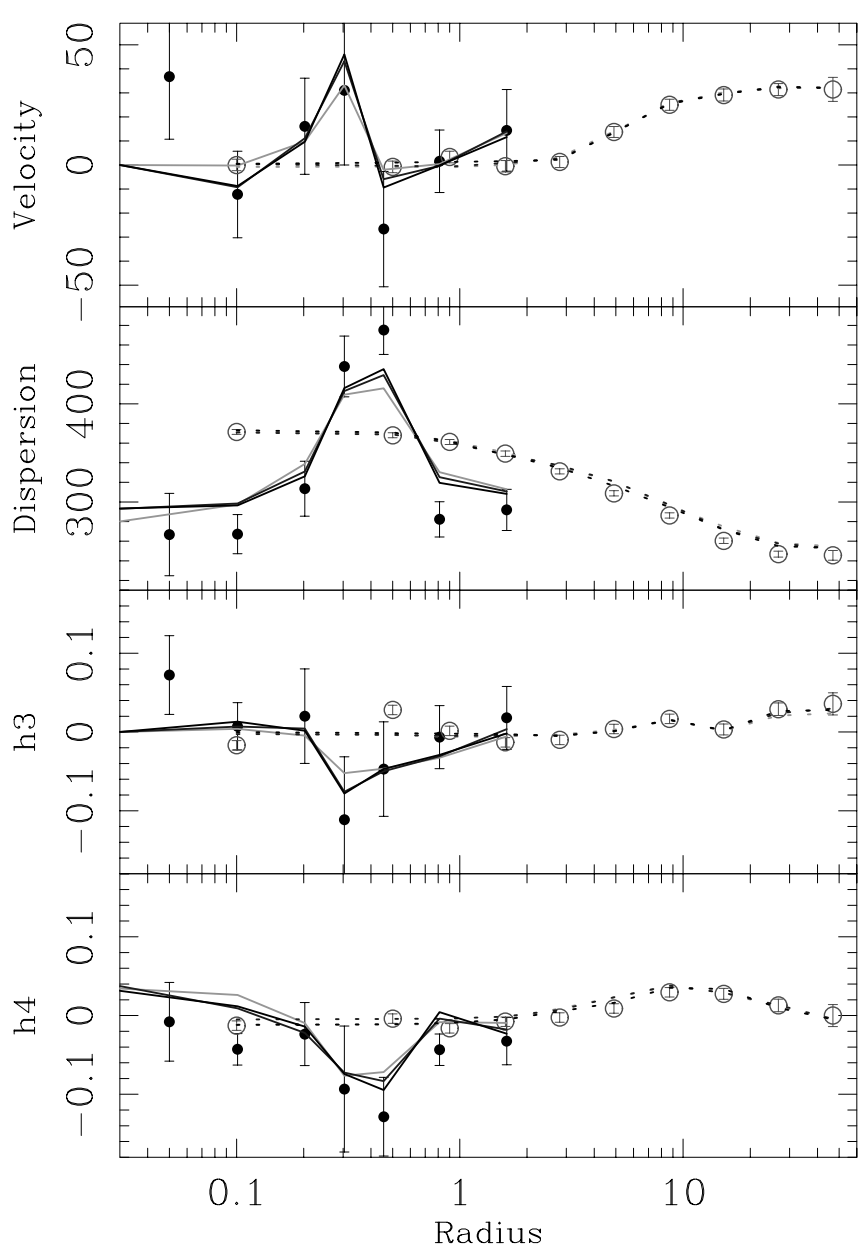

FIG. 9.-Comparison of data and models for the first four Gauss-Hermite coefficients. The filled circles represent the STIS data, and the open circles are ground-based measurements. The lines are from three different models, with the solid line for the STIS data and the dashed line for ground-based. The black lines are from the best-fit model, the light gray line is for the no black hole case, and the dark gray line is for a mass that is twice the best-fit mass. The dynamical models are fitted to the LOSVDs directly, so the comparison with the GaussHermite is only to provide a visual inspection of how well we fit the data. [See the electronic edition of the Journal for a color version of this figure.]

\section{UNCERTAINTIES FROM THE ORBIT-BASED MODELS}

For NGC 1399, we measure the black hole mass with $14 \%$ accuracy. Houghton et al. (2006) present dynamical models for NGC 1399 based on kinematics obtained on the VLT with AO. Using orbit superposition models, they find a black hole mass of $1.2(+0.5,-0.6) \times 10^{9} M_{\odot}$, a $50 \%$ accuracy. They also find strong tangentially biased orbits in the central regions, which is very similar to what we find (as plotted in Fig. 9). Statistically, there is no concern, since the two black hole masses are different by only $1 \sigma$. In fact, the Houghton et al. mass is consistent with zero at $2 \sigma$, so any black hole mass that we measure would be consistent. The question, however, is why we provide an uncertainty that is nearly 10 times smaller than what they find. The answer is most likely a combination of the data quality and differences in the dynamical modeling, which we describe below.

A similar comparison of the uncertainties can be made for other galaxies with black hole mass estimates. The published uncertainties range from $10 \%$ to over $50 \%$ (e.g., Tremaine et al. 2002), with most of these based on orbit-based models from two groups (the Nuker and Leiden groups). However, Valluri et al. (2004) and Houghton et al. (2006) both use orbit-based models and find substantially larger uncertainties for the particular galaxies 

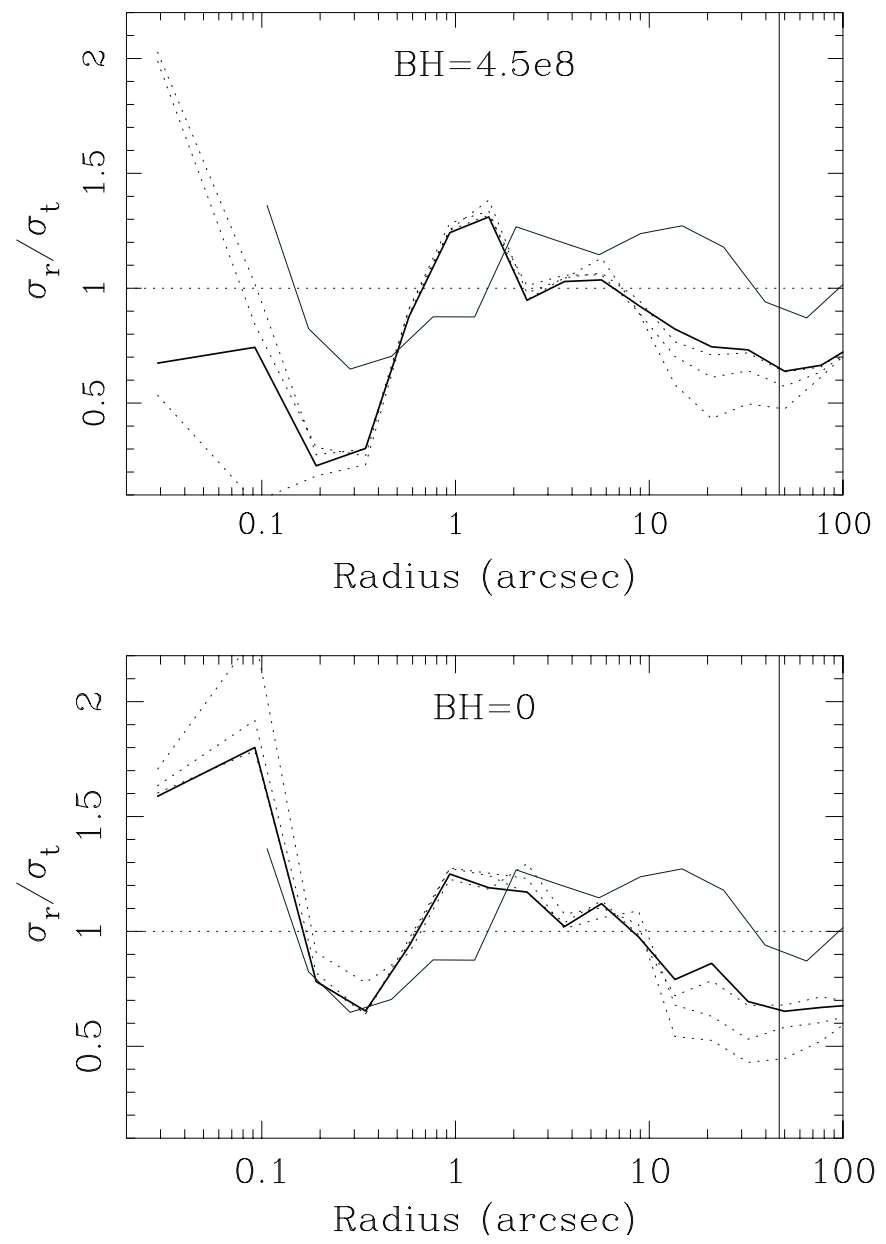

FIG. 10.- Ratio of the radial to tangential second moment of the velocity distribution for the best-fit black hole model (top) and the zero black hole model (bottom). The solid line is along the major axis, for which we have data. The dotted lines are along the other four position angles in the model, for which we do not have data. We have defined $\sigma_{t}=\left[\left(\sigma_{\theta}^{2}+\sigma_{\phi}^{2}\right) / 2\right]^{1 / 2}$, where $\theta$ and $\phi$ are the standard spherical coordinates. Our best-fit model is the top panel. The solid gray line is the ratio from Houghton et al. (2006) for their best-fit model with a black hole of $1.2 \times 10^{9} M_{\odot}$. [See the electronic edition of the Journal for a color version of this figure.]

they model. Some of this is due to the data that are being used, but part of it is in the details of the dynamical models. The most rigorous tests for recovery of the black hole mass and uncertainties is in Siopis et al. (2007), where they find that when using proper observational uncertainties, the orbit-based models provide robust estimates of the black hole mass and the uncertainties (also shown in Gebhardt 2004). As an example, one can compare the black hole mass uncertainty for two of the best measured stellar dynamical cases, our Galaxy and M32. Summarized in Ghez et al. (2005) and Schodel et al. (2003), the black hole mass in our Galaxy is known to $3 \%-6 \%$, just over a factor of 2 better than what we find in NGC 1399. Even though the Galaxy black hole is significantly more spatially resolved compared to NGC 1399, the uncertainty is driven by the small number of stars with either radial velocities or proper motions, whereas the $\mathrm{S} / \mathrm{N}$ of the central NGC 1399 is high enough that the uncertainty is driven mainly by the spatial resolution. Thus, the relative accuracy of the black hole masses is consistent. For M32, Verolme et al. (2002) measure the black hole mass to $20 \%$ accuracy for 3 degrees of freedom, and about $10 \%$ when using similar statistics as used for NGC 1399 (1 degree of freedom and marginalizing over the other parameters). Given the relative distances, black hole masses, and velocity dispersions, the on-sky black hole sphere of influence in M32 is about 2 times smaller than in NGC 1399. Thus, the relative accuracies of the black hole masses in this case are consistent as well.

However, there is a significant inconsistency with the uncertainty measured here and in Houghton et al. (2006) for NGC 1399. For our observations, the point-spread function (PSF) of STIS is well represented by an Airy function with most of the power in a single Gaussian with FWHM $=0.07^{\prime \prime}$. Our central spectral element for NGC 1399 is a $0.2^{\prime \prime} \times 0.1^{\prime \prime}$ box. The PSF of the AO data from Houghton et al. is complicated, and they represent it as a double Gaussian, with $30 \%$ of the light in a central Gaussian of FHWM $=0.15^{\prime \prime}$ (i.e., a strehl of 0.30). Furthermore, their PSF is simulated, since the star they use to provide the $\mathrm{AO}$ correction is $18^{\prime \prime}$ away from the center of NGC 1399 , but this probably only adds a small additional uncertainty on the PSF. Their slit is $0.17^{\prime \prime}$ wide. Convolving both central spectral elements with the PSF shows that the STIS data are about $50 \%$ better than the AO data in terms of spatial resolution. However, the main difference is due to the low strehl ratio of the AO data. Since NGC 1399 has a relatively flat core, the $30 \%$ strehl causes light from larger radii to have a significant contribution to the central spectral element. This effect is taken into account in their modeling. Given the better PSF and strehl of STIS, the uncertainties on the black hole are better by an appreciable amount. The other main observational difference is the spectral range. The STIS data use the $\mathrm{H}$ and $\mathrm{K}$ and G-band regions, and Houghton et al. use the $\mathrm{CO}$ band head at $2.3 \mu \mathrm{m}$. Silge \& Gebhardt (2003) show the complications that arise when using the band head, and that the main effect is to limit the accuracy of the LOSVD. Whether this effect is part of the difference in the black hole accuracy is difficult to ascertain, but could potentially be important.

The other important difference is the approach of the dynamical models. We both use orbit-based models, but we fit the LOSVD bins and they fit basis functions as a representation of the LOSVD. The advantage of the basis function is that they are mathematically uncorrelated, and the LOSVD bins are correlated. This may have some effect on the uncertainties, as discussed in Magorrian (2006). The correlation of the LOSVD bins - and the similar correlation of Gaussian-Hermite polynomial coefficients - would affect all black hole mass uncertainties from stellar dynamics that have been published. However, Gebhardt (2004) find that the uncertainties estimated from the orbit-based models are accurate, based on bootstrap simulations. Given the intrinsic scatter in black hole mass correlations to host properties is close to zero, increasing the mass uncertainties will push the intrinsic scatter to yet smaller values. For NGC 1399, the differences in data quality appear to be responsible for the difference in black hole mass uncertainty. However, resolution of this difference in the modeling approach will likely require a reanalysis of some of the data and models.

\section{DISCUSSION}

We have carefully examined the morphology around the radius where the tangential orbits dominate, but we find no obvious feature. There are no changes in the surface brightness profile, the color profile, and the ellipticity profile. A possible explanation for the tangential orbits could have been a torus of material, as has been proposed to explain hollow core galaxies (Lauer et al. 2002). A torus would also manifest itself in the orbital structure in the offset axes. Since the orbital structure appears to be similar along all position angles, we argue that the tangential structure is independent of angle. Furthermore, there is no net streaming motion measured in the LOSVD, which argues that a disk is not 
the explanation. More likely, the cause could simply be a lack of radial orbits or an enhancement of tangential orbits.

Using an integrated velocity dispersion of $337 \mathrm{~km} \mathrm{~s}^{-1}$, the black hole mass in NGC 1399 is a factor of 2.5 below that expected from the BH- $\sigma$ correlation, and a factor of 2.0 below that expected from the correlation with luminosity (Lauer et al. 2007). Saglia et al. (2000), with much worse spatial resolution, find an upper limit on the black hole mass that is consistent with our mass. It is possible that the tangential orbits and the low black hole mass are related. NGC 1399 does inhabit a special environment by being at the center of the Fornax Cluster. Whether more frequent accretion and mergers play a role in shaping its black hole mass is unknown, and it would be worthwhile to test whether binary black hole interactions could cause both the tangential orbits and relatively low black hole mass. However, the low black hole mass could reflect the intrinsic scatter in the BH- $\sigma$ correlation, with NGC 1399 being near the bottom edge of the observed scatter.

A possible scenario is to have a stellar cluster fall into NGC 1399 on essentially a purely radial orbit. In this case the cluster hits the black hole head-on and an equal number of stars pass to one side and the other side, causing no net rotation. However, this would cause there to be a preferred axis for the tangential orbits, and we see it independent of angle. If, however, the stellar cluster is quite large (i.e., around the size of the region of tangential anisotropy), then the stars should distribute themselves in a spherical pattern. Those stars that get near to the black hole - the ones on radial orbits - tend to be ejected or accreted, leaving dominance of tangential orbits. However, the extreme amount of tangential orbits in NGC 1399 needs to be compared to a detailed simulation.

We are grateful for the hospitality provided by the Observatories of the Carnegie Institute of Washington. K. G. is grateful to Andreas Burkert for stimulating discussions on the nature of the tangential orbits, and to Jens Thomas for numerous discussion of the modeling code. K. G. gratefully acknowledges NSF CAREER grant AST 03-49095. This publication is based on observations made with the NASA/ESA Hubble Space Telescope, which is operated by the Association of Universities for Research in Astronomy, Inc., under NASA contract NAS 5-26555. Financial support was provided by NASA grants GO-5990 and GO-8214 from the Space Telescope Science Institute.
Barth, A., Ho, L. C., \& Sargent, W. 2002, ApJ, 566, L13

Bender, R. 1990, A\&A, 229, 441

Cappellari, M., et al. 2006, MNRAS, 366, 1126

Cretton, N., de Zeeuw, P. T., van der Marel, R. P., \& Rix, H.-W. 1999, ApJS, 124, 383

Dressler, A. 1984, ApJ, 281, 512 1989, in IAU Symp. 134, Active Galactic Nuclei, ed. D. E. Osterbrock \& J. S. Miller (Dordrecht: Kluwer), 217

Fabian, A. 1999, MNRAS, 308, L39

Ferrarese, L., \& Merritt, D. 2000, ApJ, 539, L9

Gebhardt, K. 2004, in Coevolution of Black Holes and Galaxies, ed. L. C. Ho (Pasadena: Carnegie Obs.), 248

Gebhardt, K., et al. 1996, AJ, 112, 105 2000, ApJ, 539, L13 2003, ApJ, 583, 92

Ghez, A., et al. 2005, ApJ, 620, 744

Graham, A., Colless, M., Busarello, G., Zaggia, S., \& Longo, G. 1998, A\&AS, 133,325

Houghton, R., Magorrian, J., Sarzi, M., Thatte, N., Davies, R., \& Krajnovic, D. 2006, MNRAS, 367, 2

Kormendy, J. 1993, in The Nearest Active Galaxies, ed. J. Beckman, L. Colina, \& H. Netzer (Madrid: CSIC), 197

Kormendy, J., \& Richstone, D. 1995, ARA\&A, 33, 581

Lauer, T., et al. 2002, AJ, 124, 1975

2005, AJ, 129, 2138

\section{REFERENCES}

Lauer, T., et al. 2007, ApJ, 662, 808

Leitherer, C., et al. 1996, PASP, 108, 996

Magorrian, J. 2006, MNRAS, 373, 425

Magorrian, J., et al. 1998, AJ, 115, 2285

Pinkney, J., et al. 2003, ApJ, 596, 903

Robertson, B., Hernquist, L., Cox, T., Di Matteo, T., Hopkins, P., Martini, P., \& Springel, V. 2006, ApJ, 641, 90

Saglia, R. P., Kronawitter, A., Gerhard, O., \& Bender, R. 2000, AJ, 119, 153

Schodel, R., Ott, T., Genzel, R., Eckart, A., Mouawad, N., \& Alexander, T. 2003, ApJ, 596, 1015

Silge, J., \& Gebhardt, K. 2003, AJ, 125, 2809

Silk, J., \& Rees, M. J. 1998, A\&A, 331, L1

Siopis, C., et al. 2007, ApJ, submitted

Springel, V., Di Matteo, T., \& Hernquist, L. 2005, MNRAS, 361, 776

Thomas, J., Saglia, R., Bender, R., Thomas, D., Gebhardt, K., Magorrian, J., Corsini, E., \& Wegner, G. 2005, MNRAS, 360, 1355

Thomas, J., Saglia, R., Bender, R., Thomas, D., Gebhardt, K., Magorrian, J., \& Richstone, D. 2004, MNRAS, 353, 391

Tonry, J. L., Dressler, A., Blakeslee, J. P., Ajhar, E. A., Fletcher, A. B., Luppino, G. A., Metzger, M. R., \& Moore, C. B. 2001, ApJ, 546, 681 Tremaine, S., et al. 2002, ApJ, 574, 740

Valluri, M., Merritt, D., \& Emsellem, E. 2004, ApJ, 602, 66

Verolme, E., et al. 2002, MNRAS, 335, 517

Woodgate, B., et al. 1998, PASP, 110, 1183 